\title{
Automatic identification of variables in epidemiological datasets using logic regression
}

\author{
Matthias W. Lorenz ${ }^{1 *}$, Negin Ashtiani Abdi², Frank Scheckenbach ${ }^{1}$, Anja Pflug ${ }^{1}$, Alpaslan Bülbül', \\ Alberico L. Catapano ${ }^{3,4}$, Stefan Agewall ${ }^{5,6}$, Marat Ezhov ${ }^{7}$, Michiel L. Bots ${ }^{8,9}$, Stefan Kiechl ${ }^{10}$, Andreas Orth ${ }^{2}$ \\ on behalf of the PROG-IMT study group
}

\begin{abstract}
Background: For an individual participant data (IPD) meta-analysis, multiple datasets must be transformed in a consistent format, e.g. using uniform variable names. When large numbers of datasets have to be processed, this can be a time-consuming and error-prone task. Automated or semi-automated identification of variables can help to reduce the workload and improve the data quality. For semi-automation high sensitivity in the recognition of matching variables is particularly important, because it allows creating software which for a target variable presents a choice of source variables, from which a user can choose the matching one, with only low risk of having missed a correct source variable.
\end{abstract}

Methods: For each variable in a set of target variables, a number of simple rules were manually created. With logic regression, an optimal Boolean combination of these rules was searched for every target variable, using a random subset of a large database of epidemiological and clinical cohort data (construction subset). In a second subset of this database (validation subset), this optimal combination rules were validated.

Results: In the construction sample, 41 target variables were allocated on average with a positive predictive value (PPV) of 34\%, and a negative predictive value (NPV) of $95 \%$. In the validation sample, PPV was $33 \%$, whereas NPV remained at $94 \%$. In the construction sample, PPV was $50 \%$ or less in $63 \%$ of all variables, in the validation sample in $71 \%$ of all variables.

Conclusions: We demonstrated that the application of logic regression in a complex data management task in large epidemiological IPD meta-analyses is feasible. However, the performance of the algorithm is poor, which may require backup strategies.

Keywords: Meta-analysis, Data management, Logic regression, Epidemiology

\section{Background}

Today, many scientific insights are gained with metaanalyses, rather than with single studies or trials, which is illustrated with raising numbers of publications based on meta-analyses. Individual participant data (IPD) metaanalyses are far less frequent, but increasing steeply as well. Depending on the scientific question, IPD metaanalyses are superior to publication-based meta-analyses

\footnotetext{
* Correspondence: Matthias.lorenz@em.uni-frankfurt.de

'Department of Neurology, University Clinic Frankfurt, Schleusenweg 2-16,

D-60528 Frankfurt/Main, Germany

Full list of author information is available at the end of the article
}

in many aspects, including the possibility to choose uniform statistical models with uniform adjustment, and-if the search is systematic-a better control of publication bias [1]. Prospectively planned pooled analyses-however optimal [1] - are still very rare, given the unproportional higher organisational effort needed.

Of course, the conduct of an IPD meta-analysis is far more laborious than a publication-based one. One large part of the workload is the harmonization of the acquired datasets. To facilitate the statistical analysis, all datasets must be transformed in a consistent format, which includes using uniform variable names and coding. In a 
large number of cohorts, that were planned and designed independently, the retrospective harmonization of the resulting data can become an immensely complex task $[2,3]$. Furthermore, manual serial harmonization of many datasets is dull work that is prone to errors that have the potential to compromise the integrity of the meta-analysis [4]. Automated identification of variables might help to reduce the load of monotonous work, and therefore capacitates the data manager to put maximal focus on data quality [4].

The PROG-IMT project (Individual progression of carotid intima media thickness as a surrogate for vascular risk) is a large IPD meta-analysis project, with the aim to assess whether the annual change of intima media thickness (IMT, a high-resolution ultrasound measure within the carotid artery wall) is a surrogate for clinical endpoints, like myocardial infarction, stroke, or death. The project works in three stages, where a large number of datasets have been acquired, and their number is steadily growing. Details of the project plan have been published in a rationale paper [5]. The acquired datasets stem from large epidemiological population studies, from hospital cohorts and from randomized clinical trials (RCTs), each comprising between 200 and 2000 variables and between 100 and 15,000 participants. They have in common that the same set of variables is used for statistical analysis, including demographic data, vascular risk factors, and IMT. When the current project was started, we expected to acquire up to 250 individual participant datasets in heterogeneous format and coding.

In order to design a computer program that helps to reduce the workload of dataset harmonization, the first step is to find criteria to assign the correct source variable to a specific target variable in the created uniform dataset ('allocation'). This can be attempted with simple rules, like < 'cholesterol' in 'variable name' indicates the target variable 'total cholesterol' $>$; or $\langle$ a median value greater than 94 indicates the target variable 'systolic blood pressure'>. To obtain reliable performance, several of these rules have to be combined.

Logic regression is a relatively new statistical method that enables to combine simple binary rules in complex logic trees, and that provides methods to find optimal Boolean combinations [6]. As yet, this method has mostly been used in genetics [7-11] and oncology [12] to optimize complex models for disease prediction; to the best of our knowledge it hasn't been applied to data management problems. Aim of this study was to apply logic regression techniques to the problem of assigning variables, as explained above, and to validate the performance of this approach, using data from the PROG-IMT project.

\section{Methods}

The PROG-IMT project is involved in using datasets from population-based epidemiologic studies, from risk populations and from RCTs. At the time these analyses were started, 34 datasets were available that were already manually harmonized. These were randomly (1:1) assigned to a construction subset, or a validation subset (Table 1). All these datasets include many variables; some of those correspond to predefined target variables, which are needed for the statistical analysis of the main project. This set of target variables is shown in Table 2. The overall algorithm followed is shown graphically in Fig. 1.

In a first step, a set of simple rules was manually created (four to 41) for every target variable, by an epidemiologist experienced in the handling of data of this type. These rules are described in Additional file 1: Table S1. These rules included conditions on the variable name, the variable label, variable type (number, date or string), scale level (ratio, ordinal or nominal, dichotomous nominal); in nominal or ordinal variables the number of values and the proportion of the most frequent value; and in ratio variables the median and the interquartile range.

For rules that involved a cutoff value (eg. median greater than 44), this cutoff was optimized with ROC analysis, with the aim to maximize the expression 'sensitivity + specificity'. For every target variable, logic regression models were created by Boolean combination of the specific rules, or a subset of these. To find an optimal Boolean combination of rules (example in Fig. 1), we applied the 'simulated annealing' algorithm [4].

Simulated annealing is a generic optimization procedure commonly used to optimize non-convex optimization problems. It presupposes that an application specific score or evaluation or loss function has been defined which assigns a penalty to each state of a system. Simulated annealing then iteratively perturbs the system using applications specific basic operations, in this case tree pruning manipulations as mentioned below, with the aim of reducing the score value of the perturbed state. The perturbations are chosen in a random way with state transition probabilities changing in the course of the iteration. This lowering of transition probabilities is the analogue of lowering of temperature in random motion in physical science and is the basic mechanism in simulated annealing to reduce the danger of missing the global optima, while at the same time allowing for convergence of the iteration. In the current work transition probabilities were systematically reduced from 0.1 to 0.0001 . When using simulated annealing for logic regression in the context of identifying source variable names, the states of the system are logical expressions, like for example $\left(R_{1} \vee R_{2}\right) \quad R_{3}$ that assign a true or false value to candidate variable name based on the rules $R_{1}, R_{2}, R_{3}$. The evaluation function was a weighted least squares function of the type $S W S_{\text {res }}=\Sigma w_{i}$ $\left(y_{i}-y_{i, p r e d}\right)^{2}$, which in the case of classification, where $y_{i}$ and $y_{i, p r e d}$ are 0 or 1 , is just a weighted misclassification 
Table 1 Datasets used for construction and validation

\begin{tabular}{|c|c|c|c|c|c|}
\hline Acronym or designation & Study name & Study type & $\begin{array}{l}\text { Number of } \\
\text { variables }\end{array}$ & $\begin{array}{l}\text { Number of } \\
\text { participants }\end{array}$ & Use \\
\hline AIR & Atherosclerosis and Insulin Resistance study & general population & 136 & 435 & Construction \\
\hline ARIC & Atherosclerosis risk in communities & general population & 10108 & 15042 & Validation \\
\hline BCAPS & $\begin{array}{l}\text { Beta-blocker Cholesterol-lowering Asymptomatic } \\
\text { Plaque Study }\end{array}$ & $\mathrm{RCT}$ & 134 & 1544 & Validation \\
\hline $\mathrm{BHS}$ & Bogalusa Heart Study & general population & 1220 & 1986 & Construction \\
\hline BKRE & Konyang University Hospital CIMT Registry & $\mathrm{RCT}$ & 109 & 205 & Validation \\
\hline Bruneck & Bruneck Study & general population & 141 & 821 & Validation \\
\hline CAPS & Carotid Atherosclerosis Progression Study & general population & 692 & 6972 & Construction \\
\hline $\operatorname{cccc}$ & Chin-Shan Community Cardiovascular Cohort Study & general population & 110 & 3603 & Construction \\
\hline $\mathrm{CHS}$ & Cardiovascular Health Study & general population & 1426 & 5901 & Construction \\
\hline CIMT_TIME & CIMT TIME Project & risk population & 144 & 671 & Validation \\
\hline CMCS-Beijing & Chinese Multi-provincial Cohort Study-Beijing & general population & 141 & 1324 & Construction \\
\hline CREED & $\begin{array}{l}\text { Cardiovascular Risk Extended Evaluation in } \\
\text { Dialysis patients }\end{array}$ & risk population & 53 & 138 & Construction \\
\hline DIWA & $\begin{array}{l}\text { Diabetes, Impaired glucose tolerance in Women } \\
\text { and Atherosclerosis }\end{array}$ & general population & 129 & 644 & Validation \\
\hline EAS & Edinburgh Artery Study & general population & 74 & 1593 & Construction \\
\hline Ekart et al. & None & risk population & 102 & 54 & Construction \\
\hline EPICARDIAN & $\begin{array}{l}\text { EPIdemiología CARDlovascular en los ANcianos, } \\
\text { Cardiovascular Epidemiology in the Elderly in Spain }\end{array}$ & general population & 76 & 446 & Construction \\
\hline EVA & Etude du Vieillissement Arteriel & general population & 212 & 1135 & Validation \\
\hline HD-IMT & $\begin{array}{l}\text { Carotid ultrasonographic parameters as markers of } \\
\text { atherogenesis and mortality rate in patients on } \\
\text { hemodialysis }\end{array}$ & risk population & 130 & 85 & Validation \\
\hline HOORN & The Hoorn Study & general population & 128 & 3103 & Construction \\
\hline IMPROVE & $\begin{array}{l}\text { Carotid Intima Media Thickness and IMT-Progression } \\
\text { as Predictors of Vascular Events in a High Risk } \\
\text { European Population }\end{array}$ & risk population & 103 & 3703 & Construction \\
\hline INVADE & $\begin{array}{l}\text { Interventionsprojekt zerebrovaskuläre Erkrankungen } \\
\text { und Demenz im Landkreis Ebersberg }\end{array}$ & general population & 1581 & 3365 & Validation \\
\hline Kato et al. & None & risk population & 131 & 284 & Validation \\
\hline $\mathrm{KIHD}$ & Kuopio Ischemic Heart Disease Risk Factor Study & general population & 151 & 1399 & Construction \\
\hline Landecho et al. & None & risk population & 69 & 248 & Validation \\
\hline Niguarda & Niguarda-Monzino Study & risk population & 88 & 1564 & Construction \\
\hline NOMAS/INVEST & Northern Manhattan Study & general population & 334 & 857 & Validation \\
\hline OSACA & Osaca Follow-Up Study for Carotid Atherosclerosis & risk population & 108 & 291 & Construction \\
\hline Papagianni et al. & None & risk population & 73 & 84 & Construction \\
\hline PIVUS & $\begin{array}{l}\text { Prospective Investigation of the Vasculature in } \\
\text { Uppsala Seniors }\end{array}$ & general population & 98 & 1017 & Validation \\
\hline PLIC & $\begin{array}{l}\text { Progression of Lesions in the Intima of } \\
\text { the Carotid }\end{array}$ & general population & 264 & 2607 & Validation \\
\hline RIAS & Resistive Index in AtheroSclerosis & risk population & 67 & 158 & Construction \\
\hline Rotterdam & Rotterdam Study & general population & 34 & 7983 & Validation \\
\hline SAPHIR & $\begin{array}{l}\text { Salzburg Atherosclerosis Prevention program } \\
\text { in subjects at High Individual Risk }\end{array}$ & general population & 141 & 3127 & Validation \\
\hline SHIP & Study of Health in Pomerania & general population & 320 & 4308 & Construction \\
\hline
\end{tabular}




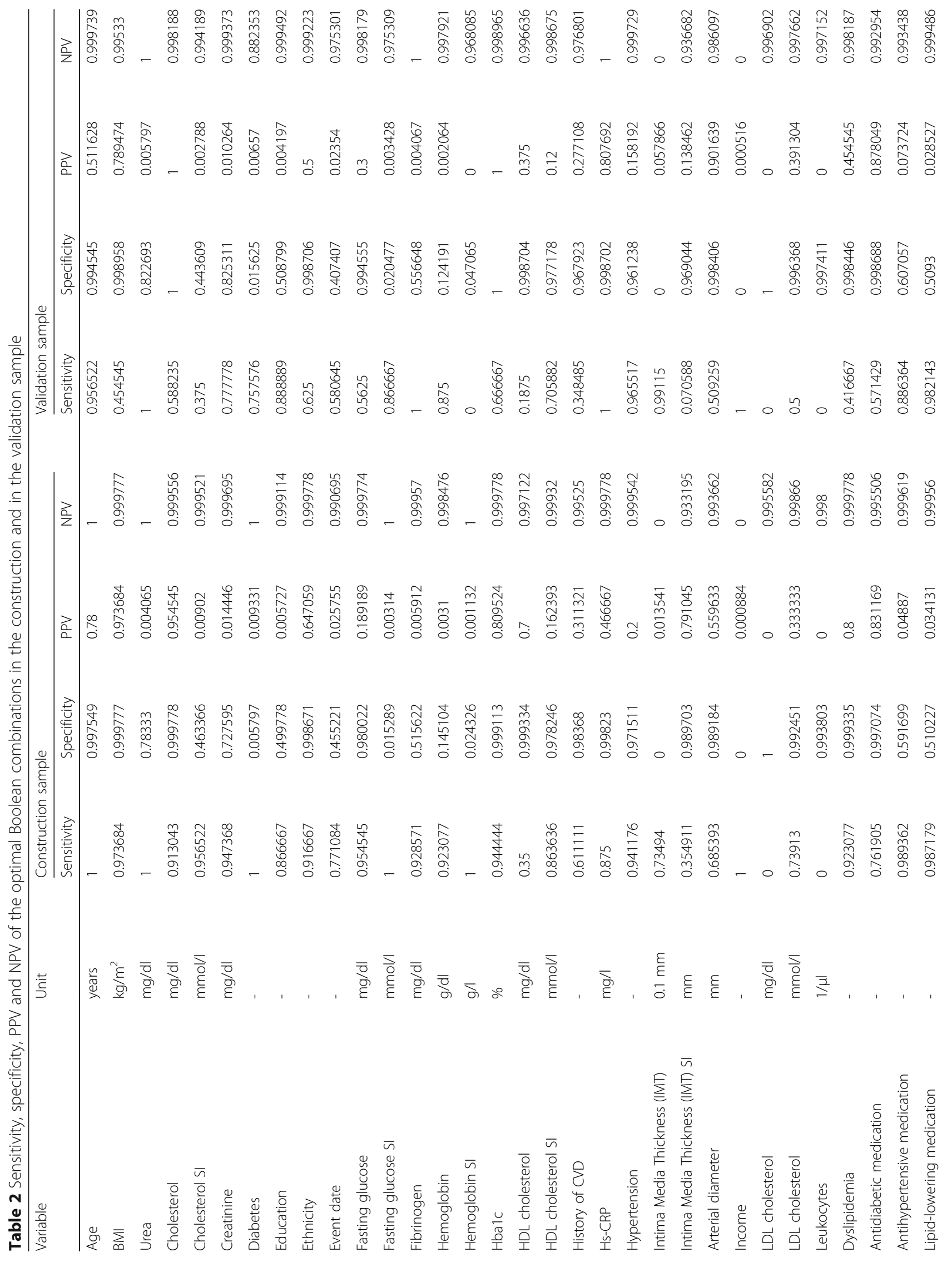




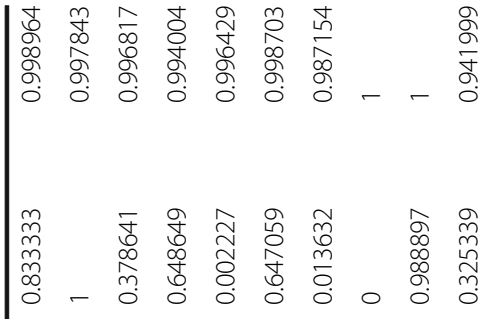

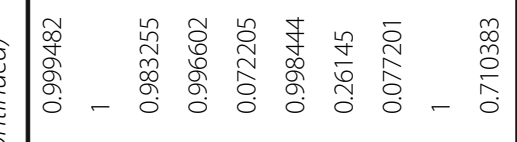

¿े

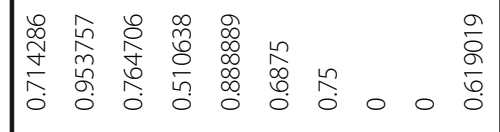

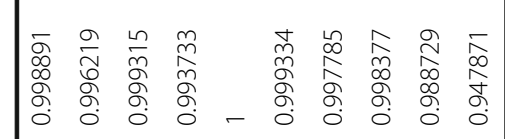

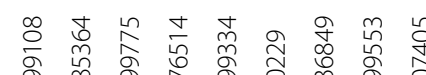

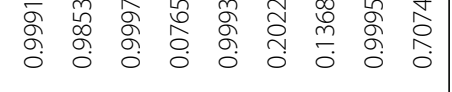
$\frac{\sqrt{0}}{\stackrel{8}{(1)}}$ 


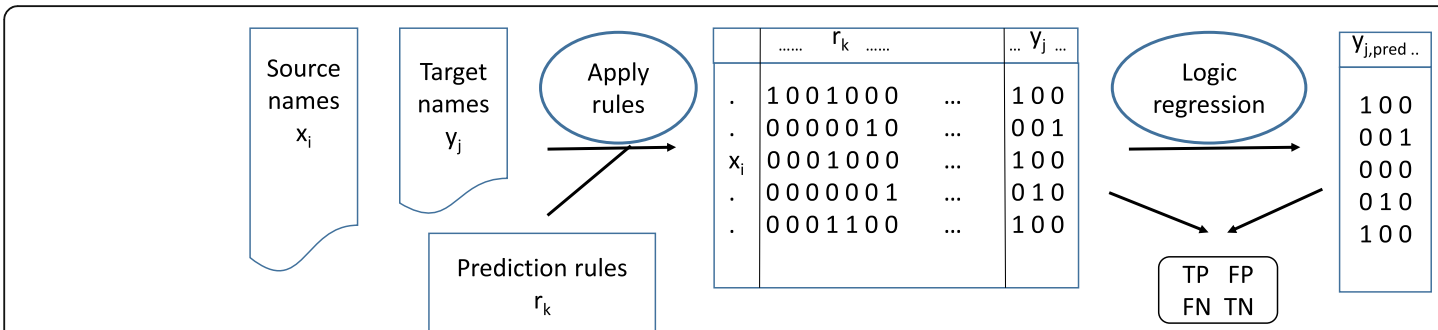

Fig. 1 Fictitious example of a logic tree combining allocation rules

count. In order to increase sensitivity without undue loss of specificity, much higher weight was given to the positives (0.9995, opposed to 0.0005 to the negatives), thus compensating the much higher number of negatives, and the basic operations are changes in the logical expression like "alternating leaves", "alternating operators", "growing a branch", "pruning a branch", "splitting a leaf" or "deleting a leaf". The names of these operations are better understood, when visualizing a logical expression as a tree.

In order to understand the dependency of sensitivity and specificity on the tuning parameters of the annealing algorithm a factor analysis was performed. Two methods were used, classification and logistic regression, four different weights for the negatives, $5^{*} 10^{-4}, 5^{*} 10^{-3}, 5^{*} 10^{-2}$, and $5^{*} 10^{-1}$, two tree sizes 5 and 10 and two values namely 4 and 8 were used for the minimum number of cases for which the tree needs to be 1 . A $2^{3} \times 4$ hybrid factorial design was performed. This yielded 32 runs for sensitivity and specificity and allowed finding interactions between the factors.

An optimization with the aim of maximizing sensitivity (low limit 99\%) and specificity (low limit 75\%) followed by dynamic profiling gave the result that direct classification is better than logistic regression and that due to the high interaction between the weights and the classification method, low weights are important to achieve high sensitivity. The loss in specificity that results from lowering the weights is less important than the gain in sensitivity (Figs. 2 and 3 ).

To find optimal combinations of rules for every target variable we used the training subset of datasets. Logic regression was applied in several models, where different configuration parameters, such as the weight of cases (matching variables) and controls (non-matching variables), and the link function itself (classification or logistic model), were varied.

After optimal configuration parameters were found, the stability of the method was tested using crossvalidation: each $10 \%$ of the data were predicted from models derived from the remaining $90 \%$ of data in turn. As it is a typical characteristic of logic regression that different source data result in qualitatively very different logic trees, these models couldn't be compared on the procedural level. Therefore we compared the resulting

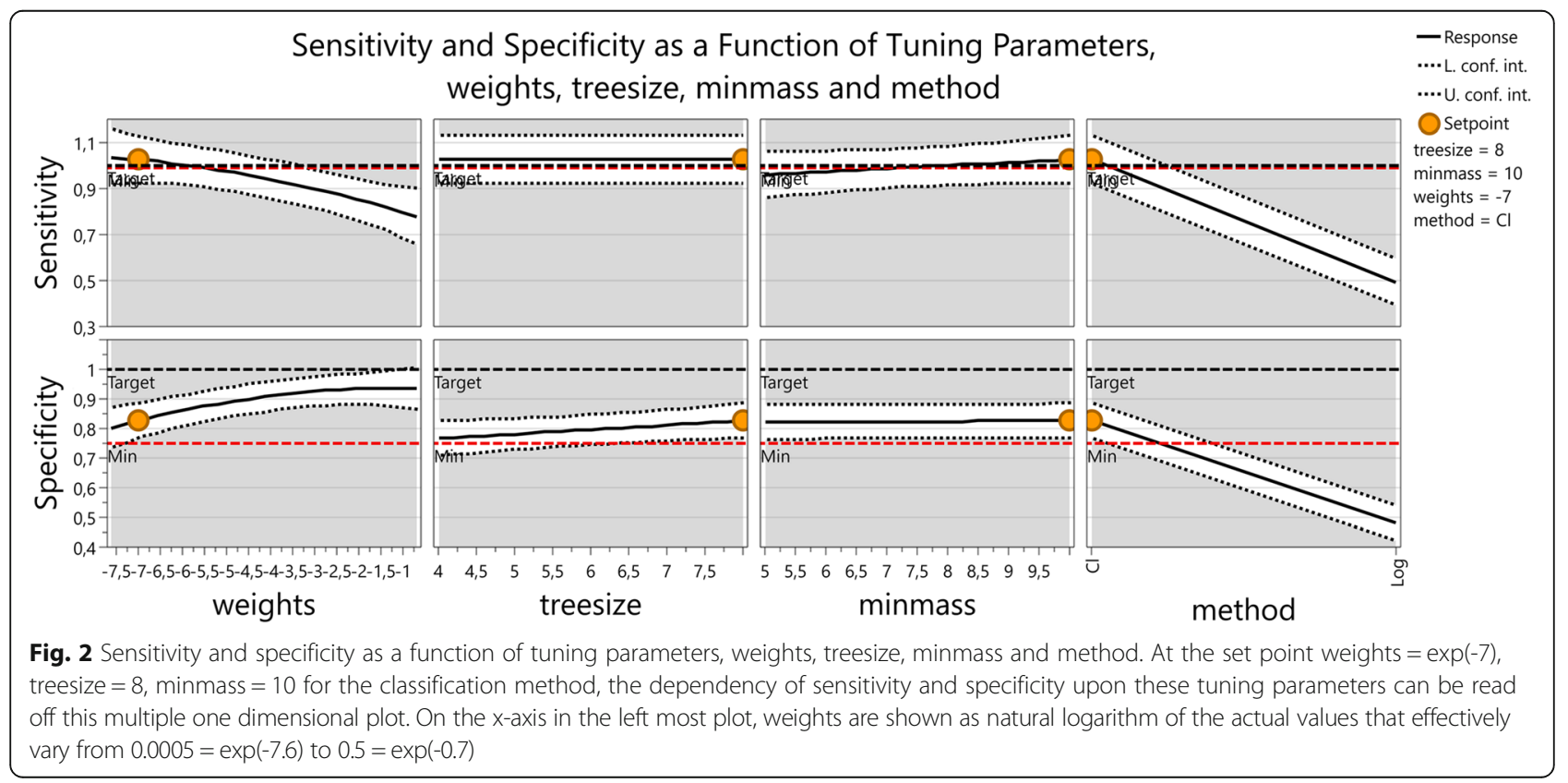




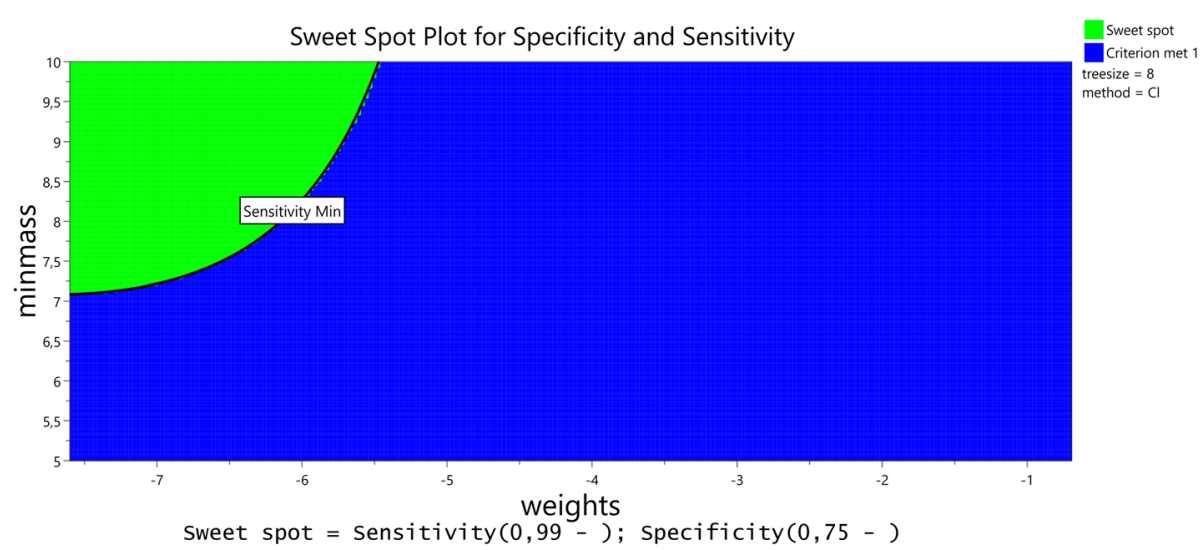

Fig. 3 Sweetspot plot for sensitivity and specificity. The same information as in Fig. 2 as a two dimensional Contour Plot (Sweet Spot Plot) for Specificity and Sensitivity. For low values of weights and high values of minmass, treesize $=8$ and the classification method, sensitivity can be raised above $99 \%$ without lowering specificity below $75 \%$. On the $x$-axis, weights are again shown as natural logarithm of the actual values

model quality in terms of sensitivity and specificity to detect a specific target variable.

The best model was fixed, and used to predict the correct assignment of variables in the validation sample. The resulting precision in the validation data was assessed using sensitivity, specificity, positive and negative predictive values. In the context of the present study, sensitivity of a target variable is the portion of matching source variables that are correctly identified. Positive predictive value (PPV) is the portion of identified source variables for which the identification is correct. Correspondingly, specificity is the portion of non-matching source variables that are identified as such and negative predictive value (NPV) is the portion of negatively identified source variables for which this identification is correct.

The source data were prepared with SAS version 9.3 (The SAS Institute, Cary, USA) and stored into a.csv file format. For the data handling and logic regression we wrote programs within C\#, using $\mathrm{R}$ and R.NET libraries, including those from the $\mathrm{R}$ software package developed by Ingo Ruczinski, Charles Kooperberg, and Michael LeBlanc at the Fred Hutchinson Cancer Research Center in Seattle (CRAN package version 3). The design for the optimization of tuning parameters and the optimization were done with MODDE Pro version 11 (mks Data Analytic Solutions, Umea, Sweden).

\section{Results}

As expected from a classification algorithm using a tree based method the logic trees themselves were quite different among different cross validation runs and due to the character of the simulated annealing algorithm even for repeated runs with the same input data. However the measured sensitivity and specificity of different runs of the algorithm were quite stable and allowed for reliable comparisons. The complete best models for every target variable are shown in Additional file 1: Table S1. Table 2 shows the performance parameters of these best models. In columns 3-6, the results in the construction sample are displayed. Sensitivity was on average reasonable high $(0.80)$, as was the specificity (0.70). The PPV was overall poor (on average 0.34), NPV was good (average 0.95). In columns $7-10$ we showed the results of independent validation (in the validation sample). Here, sensitivity was considerable less (0.62), but specificity was comparable $(0.71)$, just as PPV (0.33) and NPV (0.94).

\section{Discussion}

The performance was quite heterogeneous: in some target variables, sensitivity, specificity, PPV and NPV were very high (e.g. age, antidiabetic medication). However, many other variables showed PPV that was far too low to be useful even in the construction sample. For the intended use within a computer program to support the data manager, the performance of the models seemed reasonable at the first glance, in terms of sensitivity. However, in order to determine the correct source variable for a given target variable, the most important quality indicator is PPV, which is the portion of identified source variables for which the identification is correct. When the PPV is considered, the performance of the algorithm was much worse. In fact, the majority of variable had PPV values of $50 \%$ or less $(63 \%$ in the construction sample, $71 \%$ in the validation sample). With failure rates as high as observed in the validation sample, a fictitious computer program would have to give a list of several candidate variables rather than a single result, for each target variable. Furthermore, an escape pathway would have to be implemented for the case that the true target variable was not on the list 
suggested by the program. However, even if the algorithm can only give a 'first guess' which is correct in $50 \%$, it may reduce the workload of the data manager by nearly half.

Still, from a methodologic perspective, it is remarkable that a tree based classification method based on a random process such as the 'simulated annealing' behaves in a reproducible fashion, on the result level, i.e. regarding quality characteristics such as sensitivity and specificity. The overall performance of the optimized logic regression models in the validation sample, compared to the construction sample, is quite similar to linear regression prediction models, for example. A finding that is worth noticing is that our attempts to optimize for sensitivity were counteracted by the models. For the intended use, sensitivity is more important than specificity, and PPV is more important than NPV, as a human data manager has more difficulty reviewing many variables than a short list of candidates, as long as he or she can rely on the fact that the target variable is on this short list. Therefore, we undertook efforts to optimize the evaluation function of the algorithm for high sensitivity and high PPV. In the construction sample this worked nicely by weighting the positives by 0.9995 against 0.0005 for the negatives, i.e. a factor of 1999 , for the negatives. This improved sensitivity from 0.976 (0.995 against 0.005 , i.e. 199) to 0.99948 , while reducing specificity from 0.87 to 0.78 . Interestingly enough, as can be verified in Table 2, the same models with the same weighting turned out to be more specific than sensitive in the validation sample.

As reflected by the increase of the number of metaanalyses over time, many insights may be gained with large collaborative projects collating data from many participating cohorts in the future [13]. Although, from the methodological point of view, the best form of metaanalyses are most likely prospectively planned pooled analyses $[1,13]$, such projects are still rare. This may be due to the immense efforts and high volumes of funding they require; furthermore such enterprises take many years or even decades to complete. So in the near and intermediate future, we will most likely increasingly face the 'second best option' [1]: IPD meta-analyses that require retrospective harmonization of data [14].. Whereas some meta-analyses have developed impressively professional structures and algorithms [2-4] and the overall quality of IPD meta-analyses has improved over the last decade [15], there still remains scope for improving their processes and statistical methods $[14,15]$.

To date, the aspects that are discussed in published literature include mostly statistical modelling [15-19], sometimes screening $[15,16]$, and rarely the process of harmonization of data [2-4]. Fortier et al. [2] and Doiron et al. [3] both describe detailed algorithms for the harmonization of heterogeneous data including manual allocation of target variables. Bosch-Capblanc [4] suggested a computer program with a three-stage algorithm to detect the matching source variable for each given target variable. Compared to our algorithm, the identification criteria are less refined, and it includes alternative ways of allocating if the primary identification criteria failed. To the best of our knowledge, no publication so far has refined the allocations procedures to the extent we have. As the Bosch-Capblanc algorithm [4] focused more on the actual handling of the data, a combination of his algorithm with our allocation procedure may yield excellent results, which remains to be tested.

However, the process shown here needs relevant manual preparations before an automated or semiautomated process can start, e.g. the manual definition of target-variable rules. This preparatory work is depending on the number of target variables, whereas the work saved by automating depends on the number of datasets processed. These benchmark data have to be weighted carefully to decide whether this approach is economic. Most likely, it will be economic when many datasets are processed, and few target variables are needed. If the rule definitions might be automated, too, this might facilitate the application considerably, improve reproducibility and reduce investigator bias.

\section{Conclusions}

With the current work we demonstrated that it is in principle possible to use logic regression models with the automated 'simulated annealing' algorithm for the task of allocating variables in large datasets to specific target variables. With the performance shown in the present example, however, it would be necessary to introduce precautions in the design of a computer program, to avoid missing the true matching source variable. Such precautions may include the program suggesting a list of candidate variables rather than a single matching variable, and the option of an exit path with manual allocation. In any case, the development effort for algorithm, optimal models and a computer program is very high, and may only amortize if several hundred datasets have to be handled.

\section{Additional file}

Additional file 1: Table S1. Rules for specific target variables and their best Boolean combination. Table S2. Program parameters. List of Members of the PROG-IMT Study group. (DOC $744 \mathrm{~kb}$ )

\section{Abbreviations}

IMT: Intima media thickness; IPD: Individual participant data; NPV: Negative predictive value; PPV: Positive predictive value; PROG-IMT: The 'Individual progression of carotid intima media thickness as a surrogate for vascular risk' project; RCT: Randomized controlled trial; ROC: Receiver operating characteristic 


\section{Acknowledgements}

We thank Ingo Ruczinski, Charles Kooperberg, and Michael LeBlanc at the Fred Hutchinson Cancer Research Center in Seattle for providing the public license CRAN software package, and the related documentation.

This manuscript was prepared using a limited access dataset of the Atherosclerosis Risk In Communities (ARIC) study, obtained from the National Heart, Lung and Blood Institute (NHLBI). The ARIC study is conducted and supported by NHLBI in collaboration with the ARIC Study investigators. This manuscript does not necessarily reflect the opinions or views of the ARIC study or the NHLBI. The Bruneck study was supported by the Pustertaler Verein zur Praevention von Herz- und Hirngefaesserkrankungen,

Gesundheitsbezirk Bruneck, and the Assessorat fuer Gesundheit, Province of Bolzano, Italy. The Carotid Atherosclerosis Progression Study (CAPS) was supported by the Stiftung Deutsche Schlaganfall-Hilfe. The PLIC Study is supported by a grant from SISA Sezione Regionale Lombarda. This manuscript was prepared using data from the Cardiovascular Health Study (CHS). The research reported in this article was supported by contracts N01-HC-85079 through N01-HC-85086, N01-HC-35129, N01 HC-15103, N01 HC-55222, and U01 HL080295 from the National Heart, Lung, and Blood Institute, with additional contribution from the National Institute of Neurological Disorders and Stroke. A full list of participating CHS investigators and institutions can be found at http:// www.chs-nhlbi.org. The EVA Study was organized under an agreement between INSERM and the Merck, Sharp, and Dohme-Chibret Company. The Edinburgh Artery Study (EAS) was funded by the British Heart Foundation. The IMPROVE study was supported by the European Commission (Contract number: QLG1- CT2002- 00896), Ministero della Salute Ricerca Corrente, Italy, the Swedish HeartLung Foundation, the Swedish Research Council (projects 8691 and 0593), the Foundation for Strategic Research, the Stockholm County Council (project 562183), the Foundation for Strategic Research, the Academy of Finland (Grant \#110413) and the British Heart Foundation (RG2008/014). The INVADE study was supported by the AOK Bayern. This manuscript was prepared using data from the Northern Manhattan Study (NOMAS) and the Oral Infections, Carotid Atherosclerosis and Stroke (INVEST) study. The NOMAS is funded by the National Institute of Neurological Disorders and Stroke (NINDS) grant R37 NS 029993 and INVEST by the National Institute of Dental and Craniofacial Research (NIDCR) grant R01 DE 13094. The Rotterdam Study was supported by the Netherlands Foundation for Scientific Research (NWO), ZonMw, Vici 918-76-619. The Study of Health in Pomerania (SHIP; http://ship.community-medicine.de) is part of the Community Medicine Research net (CMR) of the University of Greifswald, Germany. Collaborators within the PROG-IMT study group: Giuseppe D. Norata, PhD ${ }^{1,2}$, Jean Philippe Empana, MD, PhD ${ }^{3}$, Hung-Ju Lin, $\mathrm{MD}^{4}$, Stela McLachlan, $\mathrm{PhD}^{5}$, Lena Bokemark, MD, $\mathrm{PhD}^{6}$, Kimmo Ronkainen, $\mathrm{MSc}^{7}$, Mauro Amato, $\mathrm{PhD}^{8}$, Ulf Schminke, MD, Prof ${ }^{9}$, Sathanur R. Srinivasan, PhD, Prof.. ${ }^{10}$, Lars Lind, MD, PhD, Prof ${ }^{11}$, Akihiko Kato, MD, Prof. ${ }^{12}$, Chrystosomos $^{2}$ Dimitriadis, MD ${ }^{13}$, Tadeusz Przewlocki, MD, PhD, Prof. ${ }^{14}$, Shuhei Okazaki, MD ${ }^{15}, C D A$ Stehouwer, MD, PhD, FESC ${ }^{16}$, Tatjana Lazarevic, MA ${ }^{17}$, Peter Willeit, PhD ${ }^{18,19}$, David N. Yanez, PhD, Assoc. Prof ${ }^{20}$, Helmuth Steinmetz, MD, Prof ${ }^{21}$, Dirk Sander, MD, Prof ${ }^{22}$, Holger Poppert, MD, PhD ${ }^{23}$, Moise Desvarieux, MD, PhD, Assoc. Prof. ${ }^{24}$, M. Arfan Ikram, MD, PhD, Assoc. Prof. ${ }^{25-27}$, Sebastjan Bevc, MD, PhD, Assist Prof ${ }^{28}$, Daniel Staub, MD, Prof. ${ }^{29}$, Cesare R. Sirtori, MD, PhD, Prof. ${ }^{30}$, Bernhard Iglseder, MD, Prof ${ }^{1,32}$ Gunnar Engström, MD, PhD, Prof. ${ }^{33}$, Giovanni Tripepi, $\mathrm{MSc}^{34}$, Oscar Beloqui, MD, $\mathrm{PhD}^{35}$, Moo-Sik Lee, MD., PhD., Prof. ${ }^{36,37}$, Alfonsa Friera, MD ${ }^{38}$, Wuxiang Xie, MD, PhD, Assist. Prof. ${ }^{39}$, Liliana Grigore, MD ${ }^{40}$, Matthieu Plichart, MD, PhD ${ }^{41}$, Ta-Chen Su, MD, PhD, Assoc. Prof. , Christine Robertson, MBChB ${ }^{5}$, Caroline Schmidt, PhD, Assoc Prof. ${ }^{42}$, Tomi-Pekka Tuomainen, MD, PhD, Prof ${ }^{7}$, Fabrizio Veglia, PhD ${ }^{8}$, Henry Völzke, MD, Prof ${ }^{43,44}$, Giel Nijpels, MD, PhD ${ }^{45,46}$, Aleksandar Jovanovic, MD, PhD, Prof ${ }^{47}$ Johann Willeit, MD, Prof. ${ }^{18}$, Ralph L. Sacco, MD, MS, Prof. ${ }^{48}$, Oscar H. Franco, MD, PhD, FESC, FFPH, Prof. ${ }^{49}$, Radovan Hojs, MD, PhD, Prof ${ }^{28,50}$, Heiko Uthoff, MD ${ }^{29}$, Bo Hedblad, MD, PhD, Prof ${ }^{33}$, Hyun Woong Park, M.D. ${ }^{36}$, Carmen Suarez, MD, PhD ${ }^{51}$, Dong Zhao, MD, PhD, Prof. . $^{39}$, Alberico Catapano, PhD, Prof. ${ }^{52,53}$, Pierre Ducimetiere, Prof. ${ }^{54}$, Kuo-Liong Chien, MD, Prof ${ }^{5}$, Jackie F. Price, MD ${ }^{5}$, Göran Bergström, MD, PhD, Prof ${ }^{6}$, Jussi Kauhanen, MD, Prof', Elena Tremoli, PhD, Prof ${ }^{3,57}$, Marcus Dörr, MD, Prof. ${ }^{58}$, Gerald Berenson, MD, Prof. ${ }^{59}$, Aikaterini Papagianni, MD, Assoc. Prof. ${ }^{13}$, Anna Kablak-Ziembicka, MD, PhD, Prof. ${ }^{14}$, Kazuo Kitagawa, MD, PhD ${ }^{60}$, Jaqueline.M. Dekker, Prof ${ }^{61}$, Radojica Stolic, MD, PhD, Prof ${ }^{17}$, Stefan Kiechl, MD, Prof ${ }^{18}$, Joseph F. Polak, MD, MPH, Proff ${ }^{62}$, Matthias Sitzer, MD, Prof. ${ }^{63}$, Horst Bickel, PhD ${ }^{64}$, Tatjana Rundek, MD, PhD, Prof. ${ }^{48}$, Albert Hofman, MD, PhD, Prof. ${ }^{25}$, Robert Ekart, MD, PhD, Assist. Prof ${ }^{65}$, Beat Frauchiger, MD, Prof. ${ }^{66}$, Samuela Castelnuovo, PhD ${ }^{67}$, Maria Rosvall, MD, PhD, Assoc. Prof.. ${ }^{68}$, Carmine Zoccali, MD, Prof. ${ }^{34}$, Manuel F Landecho, MD, PhD ${ }^{35}$, Jang-Ho Bae, MD.,PhD.,FACC. ${ }^{36,69}$, Rafael Gabriel, Prof., MD, Phd ${ }^{70}$, Jing Liu, MD, PhD, Prof. ${ }^{39}$, Damiano Baldassarre, PhD, Prof ${ }^{8}$, Maryam Kavousi, MD, PhD ${ }^{71}$.

\section{Affiliations of collaborators:}

${ }^{1}$ Dipartimento di Scienze Farmacologiche e Biomolecolari, Università degli Studi di Milano, Milan, Italy

${ }^{2}$ SISA Center for the Study of Atherosclerosis, Bassini Hospital, Cinisello Balsamo, Italy

${ }^{3}$ Paris Cardiovascular Research Centre (PARCC), University Paris Descartes, Sorbonne Paris Cité, UMR-S970, Paris, France

${ }^{4}$ Department of Internal Medicine, National Taiwan University Hospital, Taipei, Taiwan

${ }^{5}$ Centre for Population Health Sciences,Usher Institute of Population Health Sciences and Informatics, University of Edinburgh, Edinburgh, UK

${ }^{6}$ Wallenberg Laboratory for Cardiovascular Research,Institution for Medicin, Department for Molecular and Clinical Medicine,Sahlgrenska Academy, Gothenburg University, Gothenburg, Sweden

${ }^{7}$ Institute of Public Health and Clinical Nutrition, University of Eastern

Finland, Kuopio Campus, Kuopio, Finland

${ }^{8}$ Centro Cardiologico Monzino, IRCCS, Milan, Italy

${ }^{9}$ Department of Neurology, Greifswald University Clinic, Greifswald, Germany

${ }^{10}$ Center for Cardiovascular Health, Department of Epidemiology,

Biochemistry, Tulane University School of Public Health and Tropical

Medicine, New Orleans, USA

${ }^{11}$ Department of Medicine, Uppsala University, Uppsala, Sweden

12 Blood Purification Unit, Hamamatsu University Hospital, Hamamatsu, Japan

${ }^{13}$ University Department of Nephrology, Hippokration General Hospital,

Thessaloniki, Greece

${ }^{14}$ Department of Interventional Cardiology,Institute of Cardiology, Collegium Medicum Jagiellonian University, Krakow, Poland

${ }^{15}$ Department of Neurology, Osaka University Graduate School of Medicine, Osaka, Japan

${ }^{16}$ Department of Internal Medicine and Cardiovascular Research Institute Maastricht (CARIM), Maastricht University Medical Centre, Maastricht, the Netherlands

${ }^{17}$ Faculty of Medicine, University of Kragujevac, Kragujevac, Serbia

${ }^{18}$ Department of Neurology, Medical University Innsbruck, Innsbruck, Austria

${ }^{19}$ Department of Public Health and Primary Care,School of Clinical

Medicine,University of Cambridge, Cambridge, UK

${ }^{20}$ Department of Biostatistics, University of Washington, Seattle, USA

${ }^{21}$ Department of Neurology, Goethe University, Frankfurt am Main, Germany

22 Department of Neurology, Benedictus Hospital Tutzing \& Feldafing,

Feldafing, Germany

${ }^{23}$ Department of Neurology, Technische Universität München, Munich, Germany

${ }^{24}$ Department of Epidemiology,Mailman School of Public Health,Columbia

University, New York, USA

${ }^{25}$ Department of Epidemiology, Erasmus University Medical Center,

Rotterdam, the Netherlands

${ }^{26}$ Department of Neurology, Erasmus University Medical Center, Rotterdam, the Netherlands

${ }^{27}$ Department of Radiology, Erasmus University Medical Center, Rotterdam, the Netherlands

${ }^{28}$ Department of Nephrology,Clinic for Internal Medicine,University Medical Centre Maribor, Maribor, Slovenia

${ }^{29}$ Department of Angiology, University Hospital Basel, Basel, Switzerland

${ }^{30}$ Center of Dyslipidemias,Niguarda Ca' Granda Hospital, Milano, Italy

${ }^{31}$ Parcelsus Medical University, Salzburg, Austria

32 Department of Geriatric Medicine, Gemeinnützige Salzburger Landeskliniken Betriebsgesellschaft GmbH Christian-Doppler-Klinik, Salzburg, Austria

${ }^{33}$ Department of Clinical Sciences in Malmö, Lund University, Malmö, Sweden

${ }^{34}$ Clinical Epidemiology and Pathophysiology of Renal Diseases and Hypertension Unit, Institute of Physiology of the National Research Council (CNR), Reggio Calabria, Italy

${ }^{35}$ Department of Internal Medicine, University Clinic of Navarra, Navarra, Spain

${ }^{36}$ College of Medicine, Konyang University Hospital, Daejeon, Korea

${ }^{37}$ Department of Preventive Medicine, Konyang University, Daejeon, Korea

${ }^{38}$ Radiology Department,Hospital Universitario de la Princesa,Universidad

Autónoma de Madrid, Madrid, Spain

${ }^{39}$ Department of Epidemiology,Beijing Institute of Heart, Lung and Blood Vessel Diseases,Beijing Anzhen Hospital, Capital Medical University, Beijing, China

${ }^{40}$ Centro Sisa per lo Studio della Aterosclerosi, Bassini Hospital, Cinisello Balsamo, Italy

${ }^{41}$ Assistance Publique, Hôpitaux de Paris, Hôpital Broca, Paris, France 
42 Walleng Laboratory for Cardiovascular Research, University of Gothenburg, Gothenburg, Sweden

${ }^{43}$ German Center for Cardiovascular Research (DZHK),partner site Greifswald, Greifswald, Germany

${ }^{44}$ Institute for Community Medicine, SHIP/Clinical-Epidemiological Research, Greifswald, Germany

${ }^{45}$ Department of General Practice,VU University Medical Center, Amsterdam, the Netherlands

${ }^{46}$ EMGO Institute for Health and Care Research, VU University Medical

Center, Amsterdam, the Netherlands

${ }^{47}$ Faculty of Medicine, University of Prishtina, Prishtina\Kosovska Mitrovica, Serbia

${ }^{48}$ Department of Neurology,Miller School of Medicine,University of Miami,

Miami, USA

${ }^{49}$ Department of Epidemiology, Erasmus MC, University Medical Center

Rotterdam, Rotterdam, the Netherlands

${ }^{50}$ Faculty of Medicine, University of Maribor, Maribor, Slovenia

${ }^{51}$ Internal Medicine Department,Hospital Universitario de la

Princesa,Universidad Autónoma de Madrid, Madrid, Spain

${ }^{52}$ IRCSS Multimedica, Milan, Italy

${ }^{53}$ Department of Pharmacological and Biomolecular Sciences, University of Milan, Milan, Italy

${ }^{54}$ University Paris_Sud Xi, Kremlin-Bicêtre, Le Kremlin-Bicêtre, France

55 Institute of Epidemiology and Preventive Medicine,College of Public

Health,National Taiwan University, Taipei, Taiwan

${ }^{56}$ Wallenberg Laboratory for Cardiovascular Research, Sahlgrenska Academy,

Gothenburg University, Götheborg, Sweden

${ }^{57}$ Dipartimento di Scienze Farmacologiche e Biomolecolari, Università di

Milano, Milan, Italy

${ }^{58}$ Department B for Internal Medicine, University Medicine Greifswald,

Greifswald, Germany

${ }^{59}$ Department of Medicine, Pediatrics, Biochemistry, Epidemiology, Tulane University School of Medicine and School of Public Health and Tropical Medicine, New Orleans, USA

${ }^{60}$ Department of Neurology, Tokyo Women's Medical University, Tokyo, Japan

${ }^{61}$ Department of Epidemiology and Biostatistics, University Medical Center, Amsterdam, the Netherlands

${ }^{62}$ Tufts University School of Medicine, Tufts Medical Center, Boston, USA

${ }^{63}$ Department of Neuology, Klinikum Herford, Herford, Germany

${ }^{64}$ Department of Psychiatry and Psychotherapy, Technische Universität

München, Munich, Germany

${ }^{65}$ Department of Dialysis,Clinic for Internal Medicine,University Medical Centre Maribor, Maribor, Slovenia

${ }^{66}$ Department of Internal Medicine,Division of Angiology,Kantonsspital Frauenfeld, Frauenfeld, Switzerland

${ }^{67}$ Centro Dislipidemie E. Grossi Paoletti,Ospedale Ca' Granda di Niguarda, Milan, Italy

${ }^{68}$ Department of Clinicla Sciences in Malmö, Lund University, Malmö, Sweden

${ }^{69}$ Heart Center, Konyang University Hospital, Daejeon, Korea

70 Instituto de Investigación Princesa IP,Hospital Universitario de la

Princesa,Universidad Autónoma de Madrid, Madrid, Spain

${ }^{71}$ Department of Epidemiology and Biostatistics, Erasmus Medical Center,

Rotterdam, the Netherlands

\section{Funding}

The PROG-IMT project was funded by the Deutsche Forschungsgemeinschaft (DFG Lo 1569/2-1 and DFG Lo 1569/2-3).

\section{Availability of data and materials}

The datasets supporting the conclusions of this article were not made publicly available due to legal restrictions arising from the data distribution policy of the PROG-IMT collaboration, and from bilateral agreements between the collaboration and data contributors.

\section{Authors' contributions}

MWL leads the PROG-IMT project, had the idea, formulated the hypothesis, supervised the analysis, analyzed and interpreted the data, and wrote the paper. NA wrote the computer program, did the analysis of the processed data, and participated in writing the paper. FS helped preparing the datasets, formulated the single rules, and helper writing the paper. AP prepared the dataset for analysis and randomized them into construction and validations sample. In addition, she contributed ideas for the syntax of the rules and the design of a data management program discussed in this paper. She did the manual data management for some of the datasets used. AB did the manual data management for most datasets used, which was used as the gold standard of variable allocation. AC and SK delivered one of the datasets used. AC, SA, SK, MLB and ME contributed important intellectual content to the discussion of the paper. AO contributed to the design of the current study, supervised the programming, helped interpreting the results and contributed importantly to the discussion. All authors read and approved the final version of this manuscript.

\section{Authors' information}

$M W L, F S, A P, A B, A C, S A$, and $M E$ are all members of the PROG-IMT study group, an international collaboration of scientists with various expertise in neurology, cardiology, pharmacology and other medical fields, and in cardiovascular epidemiology and statistical methodology. Aim of the Study Group is to contribute to a number of unresolved questions in the investigation of cardiovascular and cerebrovascular risk, within the framework of the PROG-IMT project. Details on the project have been published earlier [2] and can be reviewed on the project website www.prog-imt.org. NA and AO are members of the Faculty of Computer Science and Engineering at the Frankfurt University of Applied Sciences, and contributed their skills in program design, programming and statistics.

\section{Competing interests}

The authors declare that they have no competing interests

\section{Consent for publication}

Not applicable.

\section{Ethics approval and consent to participate}

The present analysis has been performed in accordance with the Declaration of Helsinki. It is based on data of, and performed within the framework of the PROG-IMT project, which is an individual participant data meta-analysis on data from multiple clinical and epidemiological studies. All contributing studies collected informed consent of all participating individuals. The PROGIMT project was approved by the Ethics committee of Medical Faculty of Frankfurt University under the reference number 304/13.

\section{Publisher's Note}

Springer Nature remains neutral with regard to jurisdictional claims in published maps and institutional affiliations.

\section{Author details}

'Department of Neurology, University Clinic Frankfurt, Schleusenweg 2-16, D-60528 Frankfurt/Main, Germany. ${ }^{2}$ Faculty of Computer Science and Engineering, Frankfurt University of Applied Sciences, Frankfurt/Main, Germany. ${ }^{3}$ RCSS Multimedica, Milan, Italy. ${ }^{4}$ Department of Pharmacological and Biomolecular Sciences, University of Milan, Milan, Italy. Institute of Clinical Sciences, University of Oslo, Oslo, Norway. ${ }^{6}$ Department of Cardiology, Oslo University Hospital Ullevål, Oslo, Norway. ${ }^{7}$ Atherosclerosis Department, Cardiology Research Center, Moscow, Russia. ${ }^{8}$ University Medical Center Utrecht, Utrecht, The Netherlands. ${ }^{9}$ Department of Epidemiology and Biostatistics, Erasmus Medical Center, Rotterdam, The Netherlands. ${ }^{10}$ Department of Neurology, Medical University Innsbruck, Innsbruck, Austria.

Received: 12 May 2016 Accepted: 23 March 2017 Published online: 13 April 2017

\section{References}

1. Blettner M, Sauerbrei W, Schlehofer B, Scheuchenpflug T, Friedenreich C. Traditional reviews, meta-analyses and pooled analyses in epidemiology. Int J Epidemiol. 1999;28:1-9.

2. Fortier I, Doiron D, Little J, Ferretti V, L'Heureux F, Stolk RP, Knoppers BM, Hudson TJ, Burton PR, International Harmonization Initiative. Is rigorous retrospective harmonization possible? Application of the DataSHaPER approach across 53 large studies. Int J Epidemiol. 2011;40:1314-28.

3. Doiron D, Burton P, Marcon Y, Gaye A, Wolffenbuttel BH, Perola M, Stolk RP, Foco L, Minelli C, Waldenberger M, Holle R, Kvaløy K, Hillege HL, Tassé AM, 
Ferretti V, Fortier I. Data harmonization and federated analysis of population-based studies: the BioSHaRE project. Emerg Themes Epidemiol. 2013;10:12.

4. Bosch-Capblanch X. Harmonisation of variables names prior to conducting statistical analyses with multiple datasets: an automated approach. BMC Med Inform Decis Mak. 2011;11:33.

5. Lorenz MW, Bickel H, Bots ML, Breteler MMB, Catapano AL, Desvarieux M, Hedblad B, Iglseder B, Johnsen SH, Juraska M, Kiechl S, Mathiesen EB, Norata GD, Grigore L, Polak J, Poppert H, Rosvall M, Rundek T, Sacco RL, Sander D, Sitzer M, Steinmetz H, Stensland E, Willeit J, Witteman J, Yanez D, Thompson SG, The PROG-IMT Study Group. Individual progression of carotid intima media thickness as a surrogate for vascular risk (PROG-IMT) rationale and design of a meta-analysis project. Am Heart J. 2010;159:730-6.

6. Ruczinski I, Kooperberg C, LeBlanc M. Logic regression. J Comput Graphical Stat. 2003;12:475-511.

7. Kooperberg C, Ruczinski I. Identifying interacting SNPs using Monte Carlo logic regression. Genet Epidemiol. 2005;28:157-70.

8. Kooperberg C, Bis JC, Marciante KD, Heckbert SR, Lumley T, Psaty BM. Logic regression for analysis of the association between genetic variation in the renin-angiotensin system and myocardial infarction or stroke. Am J Epidemiol. 2007;165:334-43.

9. Dinu I, Mahasirimongkol S, Liu Q, Yanai H, Sharaf Eldin N, Kreiter E, Wu X, Jabbari S, Tokunaga K, Yasui Y. SNP-SNP interactions discovered by logic regression explain Crohn's disease genetics. PLoS One. 2012;7:e43035.

10. Sarbakhsh P, Mehrabi Y, Daneshpour MS, Zayeri F, Zarkesh M. Logic regression analysis of association of gene polymorphisms with low HDL: Tehran Lipid and Glucose Study. Gene. 2013;513:278-81.

11. Zhi S, Li Q, Yasui Y, Edge T, Topp E, Neumann NF. Assessing host-specificity of Escherichia coli using a supervised learning logic-regression-based analysis of single nucleotide polymorphisms in intergenic regions. Mol Phylogenet Evol. 2015;92:72-81.

12. Janes $\mathrm{H}$, Pepe M, Kooperberg C, Newcomb P. Identifying target populations for screening or not screening using logic regression. Stat Med. 2005;24: 1321-38.

13. Riley RD, Sauerbrei W, Altman DG. Prognostic markers in cancer: the evolution of evidence from single studies to meta-analysis, and beyond. $\mathrm{Br} \mathrm{J}$ Cancer. 2009;100:1219-29.

14. Stewart LA, Clarke M, Rovers M, Riley RD, Simmonds M, Stewart G, Tierney JF, PRISMA-IPD Development Group. Preferred reporting items for systematic review and meta-analyses of individual participant data: the PRISMA-IPD statement. JAMA. 2015;313:1657-65.

15. Simmonds M, Stewart G, Stewart L. A decade of individual participant data meta-analyses: A review of current practice. Contemp Clin Trials. 2015 Jun 17 [Epub ahead of print]

16. Boccia S, De Feo E, Galli P, Gianfagna F, Amore R, Ricciardi G. A systematic review evaluating the methodological aspects of meta-analyses of genetic association studies in cancer research. Eur J Epidemiol. 2010;25:765-75.

17. Debray TP, Moons KG, Abo-Zaid GM, Koffijberg H, Riley RD. Individual participant data meta-analysis for a binary outcome: one-stage or twostage? PLoS One. 2013;8:e60650.

18. Thomas D, Radji S, Benedetti A. Systematic review of methods for individual patient data meta- analysis with binary outcomes. BMC Med Res Methodol. 2014;14:79.

19. Ahmed I, Debray TP, Moons KG, Riley RD. Developing and validating risk prediction models in an individual participant data meta-analysis. BMC Med Res Methodol. 2014;14:3.

\section{Submit your next manuscript to BioMed Central and we will help you at every step:}

- We accept pre-submission inquiries

- Our selector tool helps you to find the most relevant journal

- We provide round the clock customer support

- Convenient online submission

- Thorough peer review

- Inclusion in PubMed and all major indexing services

- Maximum visibility for your research

Submit your manuscript at www.biomedcentral.com/submit
Biomed Central 